\title{
External apical root resorption and the release of interleukin-6 in the gingival crevucular fluid induced by a self-ligating system
}

\author{
Ryoko Kawashima-Ichinomiya ${ }^{1}$, Masaru Yamaguchi $^{1}$, Yasuhiro Tanimoto ${ }^{2 *}$, Masaki Asano ${ }^{1}$, \\ Kunihiko Yamada ${ }^{1}$, Ryo Nakajima ${ }^{1}$, Shoji Fujita ${ }^{1}$, Kazutaka Kasai ${ }^{1}$ \\ ${ }_{1}^{1}$ Department of Orthodontics, Nihon University School of Dentistry at Matsudo, Chiba, Japan \\ ${ }^{2}$ Department of Dental Biomaterials, Nihon University School of Dentistry at Matsudo, Chiba, Japan \\ Email: " yamaguchi.masaru@nihon-u.ac.jp
}

Received 16 February 2012; revised 6 March 2012; accepted 19 March 2012

\begin{abstract}
The aim of this study was to investigate the amount of external apical root resorption (EARR) and the release of interleukin (IL)-6 in the gingival crevicular fluid (GCF) in subjects treated with a low-force lowfriction system. Sixty patients were assigned to two groups of thirty patients for each: one group received treatment with self-ligating brackets and the other with conventional ligated edgewise brackets. All patients were treated with extraction of the maxillary first premolars. The EARR of the maxillary central incisors was evaluated on the periapical radiographs and cephalograms, taken before and after orthodontic treatment. The GCF was also collected non-invasively from the mesial and distal sides of central incisors by using filter paper strips before and after orthodontic treatment. Enzyme-linked immunosorbent assay (ELISA) kits were used to determine the IL-6 levels in the GCF samples. A significant difference was found in the amount of EARR between the patients with self-ligating brackets and conventional brackets. The mean amount of EARR was significantly lower for self-ligating brackets than conventional brackets $(p<0.05)$. The GCF levels of IL-6 for the patients with self-ligating brackets appliance were significantly lower than for those with the conventional brackets $(p<0.05)$. These results show that the mean amount of EARR and the GCF levels of IL-6 were significantly lower in the patients treated using low-force low-friction appliances than conventional brackets. Therefore, self-ligating brackets may be a useful system for reducing inflammation and EARR.
\end{abstract}

Keywords: IL-6; Gingival Crevucular Fluid; External Apical Root Resorption; Self-Ligating Brackets

"Corresponding author.

\section{INTRODUCTION}

External apical root resorption (EARR) is an unavoidable pathological consequence of orthodontic tooth movement. It can be defined as an iatrogenic disorder that unpredictably occurs after orthodontic treatment, whereby the resorbed apical root portion is replaced with normal bone. EARR is a sterile inflammatory process that is extremely complex, and involves various disparate components, including mechanical forces, tooth and bone, cells, the surrounding matrix, and certain known biologic messengers [1,2]. Interleukin-6 (IL-6) is a potent stimulus for bone resorption and osteoclastic cell recruitment during orthodontic tooth movement $[3,4]$. With regard to the relationship between EARR and IL-6, Hayashi et al. indicated that IL- 6 is important for the induction and further processing of mechanically-induced root resorption in the rat [5]. Yamaguchi et al. reported that Stimulation with substance P increased the levels of IL-6 in obtained from patient with severe root resorption [6]. Therefore, IL-6 may contribute to EARR during orthodontic tooth movement.

Wire friction influences the forces acting in a continuous arch system. Damon suggested that the use of a nearly friction-free system, using self-ligating brackets and high-tech wires, may not cause the periodontal problems, including alveolar bone loss, which are typically associated with orthodontic treatment [7]. Other studies have reported that the static friction measured in vitro is much lower when a passive self-ligating system is used than when any other type of fixed appliance is used $[8,9]$. The friction force disturbs orthodontic tooth movement, thus, it is expected that the influence on the periodontal tissue would be different for the self-ligating brackets compared to the conventional appliances. We previously reported that the gingival crevicular fluid (GCF) levels of substance P (SP), one of neuropeptides which cause local inflammation, for the passive self-ligating system sites were significantly lower than those for the teeth with 
conventional brackets at 24 hours after initiating treatment [10]. Therefore, the passive self-ligating system is useful to reduce the inflammation and pain resulting from orthodontic forces.

The purposes of this study were to measure and compare the EARR and the levels of IL- 6 in the GCF in patients undergoing treatment with self-ligating brackets compared with those undergoing treatment using conventional appliances.

\section{MATERIALS AND METHODS}

\subsection{Subject Selection}

Sixty subjects were selected from patients seeking treatment in the Department of Orthodontics at the Nihon University School of Dentistry at Matsudo. Sixty orthodontic patients (15 males, 45 females, mean age of 18.0 \pm 5.3 years) were enrolled in the study, after meeting the following criteria: 1) good general health; 2) lack of antibiotic therapy during the previous 6 months; 3) absence of anti-inflammatory drug administration in the month preceding the study; 4) healthy periodontal tissues with generalized probing depths $\leq 3 \mathrm{~mm}$ and no radiographic evidence of periodontal bone loss. Informed consent from the subjects was obtained after an explanation of the study protocol, which was reviewed by the ethics committee of Nihon University School of Dentistry at Matsudo (\#10-019).

Two groups were set up, one treated with "conventional brackets" (CB) and the other treated with "selfligation brackets" (SL). Thirty patients (7 males, 23 females) were treated with the self-ligating brackets (Damon 3; Ormco, Japan, Tokyo, Japan). Damon ${ }^{\circledR} 3$ passive SLBs and Damon arch wires, treatment protocol according to the Damon Workbook. A matched control group of 30 patients ( 8 males, 22 females) was selected from the same registry and treated with the conventionally ligated standard edgewise bracket system (0.022inch slot; Ormco). These controls were matched with the other group for age, sex, ANB, overjet, and overbite values before orthodontic treatment (T1).

The selection criteria for the subjects were the following: 1) a Class I dental and skeletal malocclusion with mild crowding ( $\leq 6 \mathrm{~mm}$; mean $5.4 \pm 0.55)$; 2) permanent dentition, no prior orthodontic treatment, constricted arches or crowding in both arches, and no skeletal crossbites present; 3) Four premolar extractions; 4) Excellent quality records; 5) Only patients with no history or evidence of tooth injury or wear, as shown on the charts and diagnostic records, were included.

\subsection{Measurement of the EARR and Tooth Position}

To record the EARR and tooth position parameters, the following measurements and evaluations were executed. The tooth length of the maxillary central incisor at T1 and T2 (after orthodontic treatment) was measured on the periapical radiographs and cephalograms, and from the incisal edge to the apex. When a difference in the length of the 2 adjacent maxillary central incisors was evident, the shorter root length was recorded. Baseline measurements of the ANB angle, overjet (along the occlusal line), and overbite (perpendicular to the occlusal line) at $\mathrm{T} 1$ were made on the cephalograms.

The measurements of the root length (EARR) and tooth position were performed according to the method reported by Kocadereli et al., [11] and Brin and Bollen [12]. The changes in the root length (EARR) of the maxillary central incisor was record as the difference between tooth lengths from T1 to T2. Maxillary incisor movements were measured as 1) The axial inclination of the maxillary central incisor to $\mathrm{SN}(1 / \mathrm{SN})$ between $\mathrm{T} 1$ and $\mathrm{T} 2 ; 2)$ The vertical and horizontal distances that the maxillary central incisor root was moved during orthodontic treatment.

\subsection{GCF Collection}

The GCF was collected from the mesial and distal sides of the upper central incisors. GCF sampling was performed using the method described by Yamaguchi et al. [13], and was collected before (T1) and after (T2) orthodontic treatment (Figure 1). The tooth was gently washed with water, and then the sites under study were isolated with cotton rolls (to minimize saliva contamination) and gently dried with an air syringe. Paper strips (Periopaper, Harco, Tustin, CA, USA) were carefully inserted $1 \mathrm{~mm}$ into the gingival crevice and allowed to remain there for 1 minute, after which a second strip was placed at the same site. Care was taken to avoid mechanical injury. The contents were eluted out into $1 \mathrm{x}$ phosphate buffer saline (PBS) containing $0.1 \mathrm{mM}$ phenylmethylsulphonyfluoride and stored at $-80^{\circ} \mathrm{C}$ until further processing.

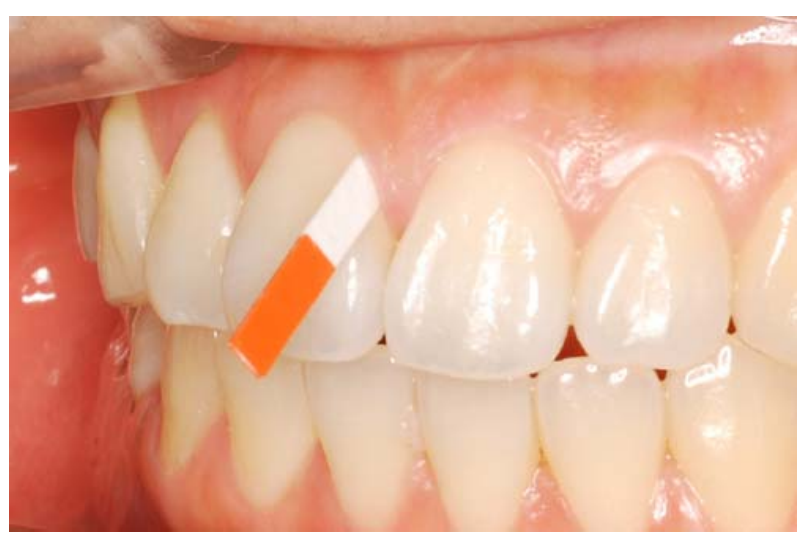

Figure 1. GCF was sampled at the mesial and distal sides of the upper central incisors. 


\subsection{Enzyme Immunoassay}

The levels of IL-6 were measured in duplicate using a commercial ELISA kit (Quantikine, R \& D Systems, Inc., Minneapolis, MN, USA), with the results expressed as $\mathrm{pg} / \mu \mathrm{g}$ of total protein in the GCF.

\subsection{Statistical Methods}

The statistical analysis of the differences among the groups was performed using a one-way ANOVA and the Scheffe test to evaluate the significance of the differences between each pair of groups.

\section{RESULTS}

The 2 groups were matched for sex and chronological age at T1. Good agreement was also found for the ANB angle, overjet and overbite at $\mathrm{T} 1$. The tooth lengths at $\mathrm{T} 1$ in both groups were similar: $26.5 \pm 1.7$ in the $\mathrm{CB}$ group and 26.6 \pm 1.9 in the SL group (Table 1).

Table 2 shows that the duration of treatment in the SL group $(25.8 \pm 3.3)$ was not significantly different from that in the CB group $(26.4 \pm 3.1)(p=0.883)$. In both groups, the lengths were reduced at T2 (Table 2): to 24.0 $\mathrm{mm} \pm 1.6$ in the CB group and $25.4 \mathrm{~mm} \pm 1.5$ in the SL group. The tooth lengths in the 2 groups were statistically

Table 1. Descriptive parameters of the 2 groups at $\mathrm{T} 1( \pm \mathrm{SD})$.

\begin{tabular}{ccc}
\hline Paremeter & CB group & SL group \\
\hline Male/female ratio & $8 / 22$ & $7 / 23$ \\
Mean age at T1 $(\mathrm{y})$ & $18.8 \pm 5.5$ & $19.0 \pm 5.3$ \\
ANB $\left({ }^{\circ}\right)$ & $3.5 \pm 1.8$ & $3.6 \pm 1.7$ \\
Overjet $(\mathrm{mm})$ & $3.6 \pm 1.7$ & $3.7 \pm 1.6$ \\
Overbite $(\mathrm{mm})$ & $3.4 \pm 1.6$ & $3.4 \pm 1.6$ \\
Crowding $(\mathrm{mm})$ & $5.4 \pm 0.55$ & $5.5 \pm 0.45$ \\
Tooth length $(\mathrm{mm})$ & $26.5 \pm 1.7$ & $26.6 \pm 1.9$ \\
\hline
\end{tabular}

Table 2. Comparison of changes ( $\pm \mathrm{SD}$ ) during mechanotherapy (T1-T2) in EARR and tooth position in the Conventional bracket and self-ligation bracket groups (absolute values in parentheses).

\begin{tabular}{cccc}
\hline & CB group & SL group & $p$ value \\
\hline Duration of trantment (mo) & $26.4 \pm 3.1$ & $25.8 \pm 3.3$ & 0.891 \\
EARR (mm) & $2.5 \pm 1.5$ & $0.88 \pm 0.9$ & $0.005^{*}$ \\
$\begin{array}{c}\text { Changes in 1/SN }\left(^{\circ}\right) \\
\text { Apex (á) vertical } \\
\text { movement (mm) }\end{array}$ & $-7.2 \pm 4.7$ & $-7.1 \pm 4.8$ & 0.901 \\
$\begin{array}{c}\text { Apex (á) horizontal } \\
\text { movement (mm) }\end{array}$ & $2.6 \pm 1.7$ & $-0.8 \pm 1.0$ & 0.883 \\
\hline
\end{tabular}

different at T2 $(p=0.05)$. The mean amount of root resorption of the maxillary central incisor as measured on the lateral cephalogram was significantly greater in the CB group than in the SL group at T2. However, this mean difference in EARR between the groups did reach statistical significance.

The axial movements of the central incisor-vertical and horizontal apical movements - are presented in Table 2 . The $1 / \mathrm{SN}$ change between $\mathrm{T} 1$ and $\mathrm{T} 2$ indicated an increase in the axial inclination in both the $\mathrm{CB}$ and SL groups (about $6^{\circ}$ ). The change in the axial inclination of the maxillary central incisor $(1 / \mathrm{SN})$ was not significantly different between the CB group and the SL group $(P=$ 0.922). The amounts of vertical movement of the apex were also not significantly difference between the groups $(P=0.931)$. For the horizontal movements of the apex, similar $(P=0.807)$ amounts of distal palatal root movement were observed in both groups.

\section{GCF STUDY}

The GCF volume has previously been shown to correlate with the inflammatory state (REF), however, there was no statistically significant difference in the mean volume of GCF between the CB group and SL group at either T1 (CB: $0.43 \pm 0.08 \mu \mathrm{l}$, SL: $0.42 \pm 0.09 \mu \mathrm{l}$ ) or T2 (CB: 0.42 $\pm 0.07 \mu \mathrm{l}, \mathrm{SL}: 0.44 \pm 0.08 \mu \mathrm{l})$ in the present study. In all of the patients, the probing depths remained less than 2 $\mathrm{mm}$ and gingival health was excellent, with no gingival bleeding.

At T1, there were no significant differences in the mean IL-6 value between the $\mathrm{CB}$ and the SL groups. However, the mean IL- 6 value in the CB group was significantly higher than that in the SL group at T2 $(p<0.05)$ (Figure 2).

\section{DISCUSSION}

In this study, Table 1 showed that the 2 groups were matched for sex and chronological age, the ANB angle, overjet and overbite, and the tooth length at T1 (Table 1). Table 2 showed that the duration of treatment, the axial movements of the central incisor in the SL group was not significantly different from that in the CB group. However, the mean amount of root resorption of the maxillary central incisor was significantly greater in the CB group (about $2.5 \mathrm{~mm}$ ) than in the SL group (about $0.9 \mathrm{~mm}$ ) at T2. This mean difference in EARR between the groups did reach statistical significance, and it may assess the significance of the stability of the tooth after orthodontic treatment. According to Weltman et al. [14], the risk factors for EARR can be divided into the treatment-related and patient-related factors. The orthodontic treatmentrelated risk factors include the treatment duration [15-17], magnitude of applied force [18-20], direction of tooth 


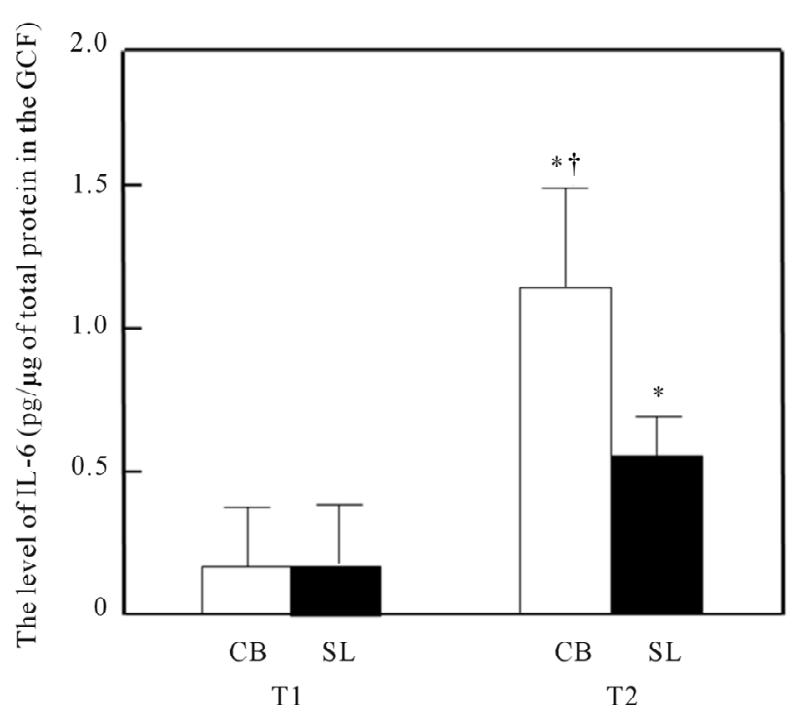

Figure 2. Changes in the IL-6 concentration in the GCF samples from the patients treated with conventional brackets (CB) and self-ligating brackets (SL). Significant differences in the concentrations between T1 and T2 are indicated with an ${ }^{*}(p<$ $0.05)$, and between the CB and SL groups with an ${ }^{\dagger}(p<0.05)$.

movement [21-23], amount of apical displacement [15, 17], and method of force application (continuous vs intermittent [24,25], type of appliance [26,27], and treatment technique) $[28,29]$.

Previous studies found that heavy forces produced significantly more EARR than light forces or controls [18-20]. Chan and Darendeliler found that the mean volume of the resorption craters was significantly (11.59 times) greater in the heavy-force group than in the control group [19]. Heavy forces in both compression and tension areas produced significantly more RR than in regions under light compression and light tension forces. Barbagallo et al. also found that heavy force produced significantly more RR ( 9 times greater than the control) than a light force (5 times greater than the control) [20]. Therefore, a light force may have an advantage for preventing the occurrence of EARR or reducing the extent of EARR.

The claim of reduced friction with self-ligating brackets is often cited as a primary advantage over the use of conventional brackets [30-32]. This occurs because the usual steel or elastomeric ligatures are not necessary, and it is claimed that passive designs generate even less friction than active ones $[33,34]$. Beger demonstrated that there was a significant decrease in the force level required for the self-ligating bracket, when compared with elastomeric and steel-tie ligation in both metal and plastic bracket systems, and concluded that self-ligating brackets require less force to produce tooth movement, leading to reduced friction [8].

GCF collection, which is a non-invasive method that has been widely used for the analysis of human tooth movement, enables the easy detection of various biochemical markers, including IL-6 [4,35]. During the process of root resorption, organic matrix proteins and cytokines are released into the gingival crevice. In this GCF study, the mean IL-6 value in the CB group was significantly higher than that in the SL at T2 $(\mathrm{p}<0.05)$ (Figure 2). George and Evans [36] demonstrated that the ratio RANKL/OPG was statistically higher in subjects with severe root resorption than in the control subjects. Therefore, GCF may be available for the study of root resorption.

The expression of IL-6 was previously shown to increase depending on the strength and duration of the compressive force in osteoblastic Saos-2 cells [37]. Lee et al. demonstrated that the IL-6 expression level from periodontal ligament (PDL) cells were increased at 72 hours after application of continuous static compressive force [38]. The concentrations of IL-6 in GCF during human orthodontic tooth movement was significantly higher at $24 \mathrm{hr}$ [35]. Moreover, IL-6 and IL-8 concentrations before orthodontic treatment were shown to be significant predictive factors for some potential inflammatory parameters during treatment [39]. Therefore, the IL-6 released in the GCF may increase depending on the strength of the orthodontic force, and the system using self-ligating brackets may inhibit the increased production of IL-6 in the GCF during orthodontic treatment. Taken together, these findings and our present results suggest that the self-ligating brackets may reduce the inflammation and EARR associated with orthodontic treatment.

Despite claims regarding the clinical superiority of selfligating brackets, evidence is generally lacking. Despite claims about the advantages of self-ligating brackets, evidence is generally lacking. Shortened chair time and slightly less incisor proclination appear to be the only significant advantages of self-ligating systems over conventional systems that are supported by the current evidence. Furthermore, Wright et al. concluded that there is no evidence that treatment with self-ligated appliances, in particular, the Damon ( $\left.{ }^{\circledR}\right)$ appliance is more stable [40]. In the presence of identical archwire sequences, there is no evidence that Damon (®) brackets can align teeth faster or in a qualitatively differently manner, when compared with conventional-ligation. There is no high quality evidence that treatment with the Damon $(\mathbb{B})$ appliance takes place more rapidly or leads to a superior occlusal or aesthetic result. Indeed, the best available evidence would suggest there is no difference in treatment outcome or time, at least in extraction cases.

In contrast to our current results, the previous studies found no significant differences in the EARR between the CB and SL groups [41,42]. Further studies in a larger 
number of patients should be carried out to investigate the advantages and disadvantages of using self-ligating brackets for orthodontic treatment.

\section{CONCLUSION}

The present results show that the mean amount of EARR and the GCF levels of IL- 6 were significantly lower in the patients treated with the low-force low-friction appliance than with conventional brackets. Therefore, selfligating brackets may be useful for reducing the inflammation and EARR associated with orthodontic treatment.

\section{ACKNOWLEDGEMENTS}

This research was supported, in part, by a Grant-in-Aid for Scientific Research from the Japan Society for the Promotion of Science (C:22592297, C:22792069, C:23792449, C:23593044).

\section{REFERENCES}

[1] Krishnan, V. and Davidovitch, Z. (2006) Cellular, molecular, and tissue-level reactions to orthodontic force. American Journal of Orthodontics and Dentofacial Orthopedics, 129, e1-e32. doi:10.1016/j.ajodo.2005.10.007

[2] Meikle, M.C. (2006) The tissue, cellular, and molecular regulation of orthodontic tooth movement: 100 years after Carl Sandstedt. European Journal of Orthodontics, 28, 221-240. doi:10.1093/ejo/cj1001

[3] Alhashimi, N., Frithiof, L., Brudvik, P., et al. (2001) Orthodontic tooth movement and de novo synthesis of proinflammatory cytokines. American Journal of Orthodontics and Dentofacial Orthopedics, 119, 307-312. doi:10.1067/mod.2001.110809

[4] Başaran, G., Ozer, T., Kaya, F.A., et al. (2006) Interleukins 2, 6, and 8 levels in human gingival sulcus during orthodontic treatment. American Journal of Orthodontics and Dentofacial Orthopedics, 130, e1-e6.

[5] Hayashi, N., Yamaguchi. M., Nakajima, R., et al. (2012) T-helper 17 cells mediate the osteo/odontoclastogenesis induced by excessive orthodontic forces. Oral Diseases, in press. doi:10.1111/j.1601-0825.2011.01886.x

[6] Yamaguchi, M., Ozawa, Y., Mishima, H., et al. (2008) Substance P increases production of proinflammatory cytokines and formation of osteoclasts in dental pulp fibroblasts in patients with severe orthodontic root resorption. American Journal of Orthodontics and Dentofacial Orthopedics, 133, 690-698.

doi:10.1016/j.ajodo.2006.03.043

[7] Damon, D.H. (1998) The Damon low-friction bracket: A biologically compatible straight-wire system. Journal of Clinical Orthodontics, 32, 670-680.

[8] Berger, J.L. (1990) The influence of the SPEED bracket's self-ligating design on force levels in tooth movement: A comparative in vitro study. American Journal of Orthodontics and Dentofacial Orthopedics, 97, 219-228.
doi:10.1016/S0889-5406(05)80055-1

[9] Sims, A.P., Waters, N.E., Birnie, D.J., et al. (1993) A comparison of the forces required to produce tooth movement in vitro using two self-ligating brackets and a pre-adjusted bracket employing two types of ligation. European Journal of Orthodontics, 5, 377-385.

[10] Yamaguchi, M., Takizawa, T., Nakajima, R., et al. (2009) The damon system and release of substance $\mathrm{p}$ in gingival crevicular fluid during orthodontic tooth movement in adults. World Journal of Orthodontics, 10, 141-146.

[11] Kocadereli, I., Yesil, T.N., Veske, P.S., et al. (2011) Apical root resorption: A prospective radiographic study of maxillary incisors. European Journal of Dentistry, 5, 318-323.

[12] Brin, I. and Bollen, A.M. (2011) External apical root resorption in patients treated by serial extractions followed by mechanotherapy. American Journal of Orthodontics and Dentofacial Orthopedics, 139, e129-e134. doi:10.1016/j.ajodo.2010.08.015

[13] Yamaguchi, M., Yoshii, M. and Kasai, K. (2006) Relationship between substance $\mathrm{P}$ and interleukin-1beta in gingival crevicular fluid during orthodontic tooth movement in adults. European Journal of Orthodontics, 28, 241-246. doi:10.1093/ejo/cji100

[14] Weltman, B., Vig, K.W., Fields, H.W., Shanker, S., Kaizar, E.E., et al. (2010) Root resorption associated with orthodontic tooth movement: A systematic review. American Journal of Orthodontics and Dentofacial Orthopedics, 37, 462-476. doi:10.1016/j.ajodo.2009.06.021

[15] Segal, G.R., Schiffman, P.H. and Tuncay, O.C. (2004) Meta analysis of the treatment-related factors of external apical root resorption. Orthodontics and Craniofacial Research, 7, 71-78. doi:10.1111/j.1601-6343.2004.00286.x

[16] Sameshima, G.T. and Sinclair, P.M. (2004) Characteristics of patients with severe root resorption. Orthodontics and Craniofacial Research, 7, 108-114. doi:10.1111/j.1601-6343.2004.00284.x

[17] Fox, N. (2005) Longer orthodontic treatment may result in greater external apical root resorption. Evidence-Based Dentistry, 6, 21. doi:10.1038/sj.ebd.6400304

[18] Harris, D.A., Jones, A.S. and Darendeliler, M.A. (2006) Physical properties of root cementum: Part 8 . Volumetric analysis of root resorption craters after application of controlled intrusive light and heavy orthodontic forces: A microcomputed tomography scan study. American Journal of Orthodontics and Dentofacial Orthopedics, 130, 639-647. doi:10.1016/j.ajodo.2005.01.029

[19] Chan, E. and Darendeliler M.A. (2006) Physical properties of root cementum: Part 7. Extent of root resorption under areas of compression and tension. American Journal of Orthodontics and Dentofacial Orthopedics, 129, 504-510. doi:10.1016/j.ajodo.2004.12.018

[20] Barbagallo, L.J., Jones, A.S., Petocz, P., et al. (2008) Physical properties of root cementum: Part 10. Comparison of the effects of invisible removable thermoplastic appliances with light and heavy orthodontic forces on premolar cementum. A microcomputed-tomography study. American Journal of Orthodontics and Dentofacial Or- 
thopedics, 133, 218-227. doi:10.1016/j.ajodo.2006.01.043

[21] Costopoulos, G. and Nanda, R. (1996) An evaluation of root resorption incident to orthodontic intrusion. American Journal of Orthodontics and Dentofacial Orthopedics, 109, 543-548. doi:10.1016/S0889-5406(96)70140-3

[22] Parker, R.J. and Harris, E.F. (1998) Directions of orthodontic tooth movements associated with external apical root resorption of the maxillary central incisor. American Journal of Orthodontics and Dentofacial Orthopedics, 114, 677-683. doi:10.1016/S0889-5406(98)70200-8

[23] Han, G., Huang, S., Von den Hoff, J.W., et al. (2005) Root resorption after orthodontic intrusion and extrusion: An intraindividual study. Angle Orthodontist, 75, 912-918.

[24] Weiland, F. (2003) Constant versus dissipating forces in orthodontics: The effect on initial tooth movement and root resorption. European Journal of Orthodontics, 25, 335-342. doi:10.1093/ejo/25.4.335

[25] Brezniak, N. and Wasserstein, A. (2002) Orthodontically induced inflammatory root resorption. Part II: The clinical aspects. Angle Orthodontist, 72, 180-184.

[26] Brezniak, N. and Wasserstein, A. (1993) Root resorption after orthodontic treatment: Part 1. Literature review. American Journal of Orthodontics and Dentofacial Orthopedics, 103, 62-66. doi:10.1016/0889-5406(93)70106-X

[27] Pandis, N., Nasika, M., Polychronopoulou, A., et al. (2008) External apical root resorption in patients treated with conventional and self-ligating brackets. American Journal of Orthodontics and Dentofacial Orthopedics, 134, 646651. doi:10.1016/j.ajodo.2007.01.032

[28] McNab, S., Battistutta, D., Taverne, A., Symons, A.L., et al. (2000) External apical root resorption following orthodontic treatment. Angle Orthodontist, 70, 227-232.

[29] Scott, P., DiBiase, A.T., Sherriff, M., Cobourne, M.T., et al. (2008) Alignment efficiency of Damon3 self-ligating and conventional orthodontic bracket systems: A randomized clinical trial. American Journal of Orthodontics and Dentofacial Orthopedics, 34, e1-e8.

[30] Chen, S.S., Greenlee, G.M., Kim, J.E., et al. (2010) Systematic review of self-ligating brackets. American Journal of Orthodontics and Dentofacial Orthopedics, 137, e1-e18. doi:10.1016/j.ajodo.2009.11.009

[31] Damon, D.H. (1998) The rationale, evolution and clinical application of the self-ligating bracket. Clinical Orthodontics and Research, 1, 52-61.

[32] Griffiths, H.S., Sherriff, M. and Ireland, A.J. (2005) Resistance to sliding with 3 types of elastomeric modules. American Journal of Orthodontics and Dentofacial Orthopedics, 127, 670-675. doi:10.1016/j.ajodo.2004.01.025
[33] Kim, T.K., Kim, K.D. and Baek, S.H. (2008) Comparison of frictional forces during the initial leveling stage in various combinations of self-ligating brackets and archwires with a custom-designed typodont system. American Journal of Orthodontics and Dentofacial Orthopedics, 133, e15-e24. doi:10.1016/j.ajodo.2007.08.013

[34] Budd, S., Daskalogiannakis, J. and Tompson, B.D. (2008) A study of the frictional characteristics of four comercially available self-ligating bracket systems. European Journal of Orthodontics, 30, 645-653. doi:10.1093/ejo/cjn058

[35] Uematsu, S., Mogi, M. and Deguchi, T. (1996) Interleukin (IL)-1 beta, IL-6, tumor necrosis factor-alpha, epidermal growth factor, and beta 2-microglobulin levels are elevated in gingival crevicular fluid during human orthodontic tooth movement. Journal of Dental Research, 75, 562-567. doi:10.1177/00220345960750010801

[36] George, A. and Evans, C.A. (2009) Detection of root resorption using dentin and bone markers. Orthodontics and Craniofacial Research, 12, 229-235. doi:10.1111/j.1601-6343.2009.01457.x

[37] Koyama, Y., Mitsui, N., Suzuki, N., et al. (2008) Effect of compressive force on the expression of inflammatory cytokines and their receptors in osteoblastic Saos-2 cells. Archives of Oral Biology, 53, 488-496. doi:10.1016/j.archoralbio.2007.12.004

[38] Lee, Y.H, Nahm, D.S, Jung, Y.K., et al. (2007) Differential gene expression of periodontal ligament cells after loading of static compressive force. Journal of Periodontology, 78, 446-452. doi:10.1902/jop.2007.060240

[39] Van Gastel, J., Teughels, W., Quirynen, M., et al. (2011) Longitudinal changes in gingival crevicular fluid after placement of fixed orthodontic appliances. American Journal of Orthodontics and Dentofacial Orthopedics, 139, 735-744. doi:10.1016/j.ajodo.2009.10.043

[40] Wright, N., Modarai, F., Cobourne, M.T., Dibiase, A.T., et al. (2011) Do you do Damon ${ }^{\circledR}$ ? What is the current evidence base underlying the philosophy of this appliance system? Journal of Orthodontics, 38, 222-230. doi: $10.1179 / 14653121141479$

[41] Pandis, N., Nasika, M., Polychronopoulou, A., Eliades, T., et al. (2008) External apical root resorption in patients treated with conventional and self-ligating brackets. American Journal of Orthodontics and Dentofacial Orthopedics, 134, 646-651. doi:10.1016/j.ajodo.2007.01.032

[42] Scott, P., DiBiase, A.T., Sherriff, M., Cobourne, M.T., et al. (2008) Alignment efficiency of Damon3 self-ligating and conventional orthodontic bracket systems: A randomized clinical trial. American Journal of Orthodontics and Dentofacial Orthopedics, 134, e1-e8. doi:10.1016/j.ajodo.2008.04.018 